\title{
An atypical component of RNA-directed DNA methylation machinery has both DNA methylation-dependent and -independent roles in locus-specific transcriptional gene silencing
}

Jun Liu ${ }^{1, *}$, Ge Bai ${ }^{2,3, *}$, Cuijun Zhang ${ }^{1, *}$, Wei Chen ${ }^{2}$, Jinxing Zhou ${ }^{1}$, Suwei Zhang ${ }^{1}$, Qing Chen ${ }^{1}$, Xin Deng ${ }^{2,4}$, Xin-Jian $\mathrm{He}^{1}$, Jian-Kang $\mathrm{Zhu}^{2}$

${ }^{1}$ National Institute of Biological Sciences, Beijing 102206, China; ${ }^{2}$ Department of Horticulture and Landscape Architecture, Purdue University, West Lafayette, IN 47907, USA; ${ }^{3}$ School of Agriculture and Biology, Shanghai Jiao Tong University, Shanghai 200240, China; ${ }^{4}$ Institute of Botany, Chinese Academy of Sciences, Beijing 100093, China

RNA-directed DNA methylation (RdDM) is an important de novo DNA methylation pathway in plants. RdDM mediates the transcriptional silencing of many endogenous genomic loci, most of which are transposon related. A forward genetics screen identified DTF1 (DNA-binding transcription factor 1) as a new component for RdDM in Arabidopsis. Loss-of-function mutations in DTF1 release the transcriptional silencing of RdDM target loci and reduce the accumulation of 24-nt small interfering RNAs (siRNAs) from some of the targets. Interestingly, in the dtf1 mutant plants, the release of transcriptional gene silencing at solo-LTR is accompanied by decreased siRNA accumulation but not by reduced DNA methylation. These results suggest that DTF1 is an atypical component of RdDM and has both DNA methylation-dependent and -independent roles in transcriptional gene silencing. We suggest that besides DNA methylation, siRNAs may cause some other uncharacterized epigenetic modifications that lead to transcriptional gene silencing.

Keywords: DTF1; siRNA; DNA methylation; transcriptional gene silencing; RdDM

Cell Research (2011) 21:1691-1700. doi:10.1038/cr.2011.173; published online 8 November 2011

\section{Introduction}

The RNA-directed DNA methylation (RdDM) and transcriptional gene-silencing pathway has been well characterized in plants [1-5]. RdDM has been shown to be required for various epigenetic processes, which include transgene silencing at the transcriptional level, genomic imprinting and suppression of retrotransposition of transposable elements [6-10]. 24-nt siRNAs and long noncoding RNAs are required for RdDM at target

*These three authors contributed equally to this work.

Correspondence: Xin-Jian He

Tel: +86-10-80707712; Fax: +86-10-80707715

E-mail: hexinjian@nibs.ac.cn

Received 15 August 2011; revised 18 September 2011; accepted 18 September 2011; published online 8 November 2011 sequences $[3,6,11]$. The plant-specific DNA-dependent RNA polymerase IV is presumably responsible for synthesizing the precursors of siRNAs [3, 12]. The precursors are converted into double-stranded RNAs by the RNA-dependent RNA polymerase RDR2 [1, 13]. The double-stranded RNA is cleaved by Dicer-like 3 into 24-nt siRNAs, which are loaded into ARGONAUTE 4 (AGO4) $[13,14]$. Another DNA-dependent RNA polymerase, Pol V, is required for producing non-coding, presumably scaffold RNAs from RdDM targets [11]. The chromatin-remodeling protein DRD1, structural-maintenance-of-chromosomes hinge domain-containing protein DMS3 and the novel plant-specific protein RDM1 form a tight protein complex (DDR), which is required for Pol V-dependent transcripts [15-18]. RDM1 is also associated with AGO4 and the DNA methytransferase DRM2 [18]. The largest subunit of Pol V, NRPE1 and the SPT5-like protein KTF1 have $\mathrm{WG} / \mathrm{GW}$ repeats in 
their C-terminal regions, which bind AGO4 and the Pol V-dependent scaffold RNAs, and help to form RdDM effector complexes $[19,20]$. The yeast IWR1-like protein RDM4/DMS4 is a transcription factor that interacts with both Pol V and Pol II. The rdm4 mutation affects Pol V-dependent transcripts and plant development $[21,22]$. DRM2 and the recently characterized DRM3 are the de novo DNA methytransferases in the Arabidopsis RdDM pathway [23-26].

siRNA-directed chromatin modification and TGS also occur in unicellular eukaryotes, such as the fission yeast [27]. Several key components of plant RdDM appear to have counterparts in fission yeast, including Argonaute AGO1, Dicer DCR1 and RNA-dependent RNA polymerase RDP1 [28]. Moreover, like the Arabidopsis WG/ GW motif-containing protein KTF1, fission yeast has a WG/GW-motif-containing protein TAS3, which is also required for interaction with Argonaute [29, 30]. The fission yeast AGO1, TAS3 and the chromodomain protein CHP1 form an RNA-induced transcriptional silencing (RITS) complex, which is essential for heterochromatin formation at pericentromeric repeats [29]. However, there is no DNA methylation in fission yeast, instead, $\mathrm{H} 3 \mathrm{~K} 9$ dimethylation catalyzed by the $\mathrm{H} 3 \mathrm{~K} 9$ methytransferase CLR4 is required for siRNA-directed repressive heterochromatin formation in fission yeast [27, 31]. In plants, $\mathrm{H} 3 \mathrm{~K} 9$ dimethylation is catalyzed by histone H3K9 methytransferases such as KYP/SUVH4 [32]. KYP forms a self-enhancing loop with the DNA methytransferase CMT3, which catalyzes DNA methylation at CHG sites [33, 34].

At some RdDM-target loci, the transcriptional gene silencing depends on MOM1 $[35,36]$. In mom 1 mutants, although the silencing of transgenes and endogenous genomic target loci is released, neither DNA methylation nor histone modification is altered at these loci [35-37]. MOM1 seems to act downstream of RdDM, although further studies are required to understand how MOM1 mediates transcriptional gene silencing independently of DNA methylation at RdDM-target loci. Interestingly, a recent study reported that MOM1 is required to prevent aberrant RNA transcriptional read-through [38]. The minimal functional domain of MOM1, CMM2, forms a coiled-coil structure, which likely interacts with other proteins $[39,40]$.

ROS1 encodes a 5-methylcytosine DNA glycosylase, which functions as a DNA demethylase by initiating a base excision repair pathway for active DNA demethylation [41-44]. ros 1 mutations cause transcriptional silencing of the transgenic $R D 29 A$ promoter-driven luciferase reporter gene $(R D 29 A-L U C)$ as well as the endogenous $R D 29 A$ gene [41]. By screening for second site sup- pressors of ros 1 mutant plants, we have identified many RdDM components [45]. Our results showed that the transcriptional silencing of the RD29A-LUC transgene in ros1 is dependent on RdDM.

In this study, we identified a novel component of RdDM, DTF1, by screening for suppressors of ros 1. dtf1 mutations release the silencing of RD29A-LUC transgene as well as the endogenous $R D 29 A$ in ros 1 . Moreover, DTF1 is required for silencing of endogenous transposable elements that are targeted by RdDM. The dtf 1 mutations cause reduced siRNA accumulation and DNA methylation at some of the RdDM targets. Surprisingly, the role of DTF1 in transcriptional silencing at some RdDM targets is not dependent on DNA methylation. These results suggest that DTF1 is an atypical RdDM component, and functions in transcriptional silencing through both DNA methylation-dependent and -independent pathways.

\section{Results}

A putative DNA-binding transcription factor, DTF1, is required for transcriptional silencing of the RD29A-LUC transgene

Our previous results showed that the RD29A promoter-driven luciferase transgene is expressed in the wildtype C24 plants under stress conditions, whereas lossof-function mutations in ROS1 result in transcriptional silencing of the RD29A-LUC transgene as well as the corresponding endogenous $R D 29 A$ [41]. RdDM is required for transcriptional silencing of transgenic $R D 29 A$ $L U C$ and endogenous RD29A in ros 1 [45]. Nearly all of the canonical RdDM components have been identified by screening for suppressors of ros 1 in our previous [45] and current studies (unpublished data). These results suggest that ROS1-mediated DNA demethylation and RdDM function antagonistically in the control of DNA methylation and transcriptional gene silencing.

Our previous ros 1 suppressor screens were carried out using an Agrobacteria-transformed population of ros $1[45,46]$. In order to identify new components of the RdDM pathway, we constructed an EMS-mutagenized library in the ros 1 mutant background that has the silenced RD29A-LUC transgene. We found most of the known RdDM components by screening for ros 1 suppressors from the library (unpublished data). In addition, we recovered a new mutant (referred to as $d t f 1$ ) where the silencing of the RD29A-LUC transgene in the ros 1 background is released.

The expression of RD29A-LUC transgene and $35 S$ promoter-driven NPTII (35S-NPTII) gene is indicated by bioluminescence intensity and kanamycin resistance, respective- 
ly $[41,46]$. In the wild-type $C 24, R D 29 A-L U C$ was highly expressed under stress conditions, and strong bioluminescence was emitted (Figure 1A). The 35S-NPTII transgene was normally expressed in the wild type, which is resistant to kanamycin (Figure 1A and 1B). ros 1 mutations cause silencing of $R D 29 A-L U C$, corresponding endogenous $R D 29 A$ and 35S-NPTII (Figure 1B) [41]. In the ros 1dtf1-1 double mutant, the silencing of RD29A-LUC and corresponding endogenous $R D 29 A$ was partially released, whereas the silencing was released in roslnrpel to a greater extent (Figure 1A and 1B). However, neither the $d t f 1-1$ nor nrpel mutation suppressed silencing of the $35 S$-NPTII transgene, which is known to be independent of RdDM (Figure 1B) [45]. The results suggest that DTF1 play an important role in transgene silencing in a manner similar to the canonical RdDM component NRPE1.

The rosldtf1-1 mutant was crossed to a homozygous ros 1 mutant (Salk_045303) in the Col-0 background, and the resulting F2 population was used for map-based cloning. The $d t f 1$ mutation was mapped to a $\sim 180 \mathrm{~kb}$ region on chromosome 1 (Figure 2A). Next-generation highthroughput DNA sequencing was carried out to sequence the whole genome of the mutant. In the $\sim 180 \mathrm{~kb}$ mapping interval, we found a $\mathrm{G}$ to A mutation in the fourth exon of At1G15215, which creates a premature stop codon (Figure 2B). The mutation was confirmed by Sanger sequencing. The results suggest that At1g15215 might be the DTF1 gene. At1g15215 encodes a putative sequencespecific DNA-binding transcription factor (hence the name DTF1, for DNA-binding transcription factor 1).

To confirm that the mutation in At1g15215 is responsible for the silencing defect in ros1dtf1-1, the genomic fragment of Atlg15215 was cloned from wild-type plants and transformed into ros 1dtf1-1 for complementation test. Compared to ros 1dtfl-1, the transgenic plants emitted much less luminescence (Figure 2C), suggesting that the silencing of RD29A-LUC transgene is restored by the wild-type transgene. In addition, we tested and found that AtSN1 methylation in ros 1dtf1 was low, but the methylation in the transgenic plants was restored (Figure 2C). Furthermore, we obtained a homozygous T-DNA insertion mutant (Salk_074540C), and found that the TDNA mutant also showed a reduction in DNA methylation at AtSN1 (Figure 2D). These results demonstrate that At1G15215 is DTF1, and is required for the silencing of RD29A-LUC transgene.
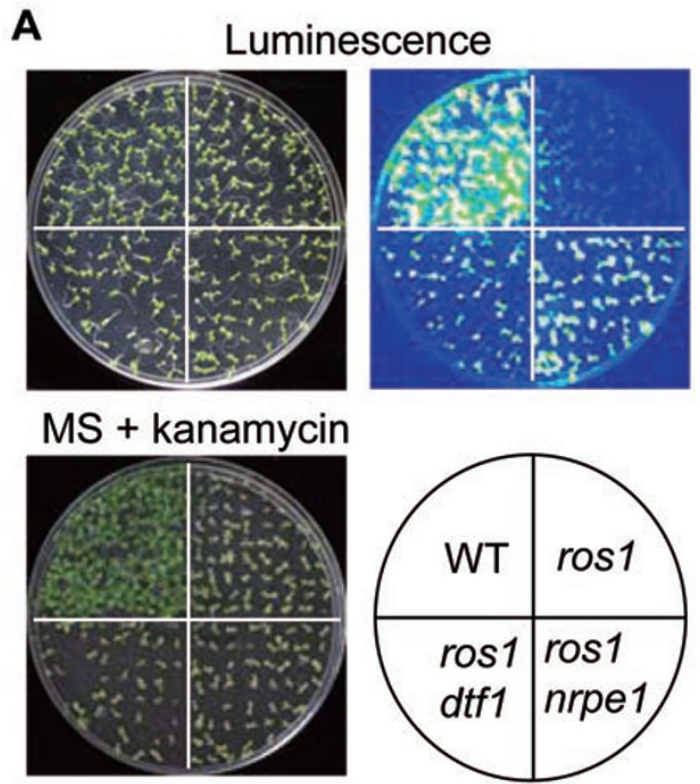

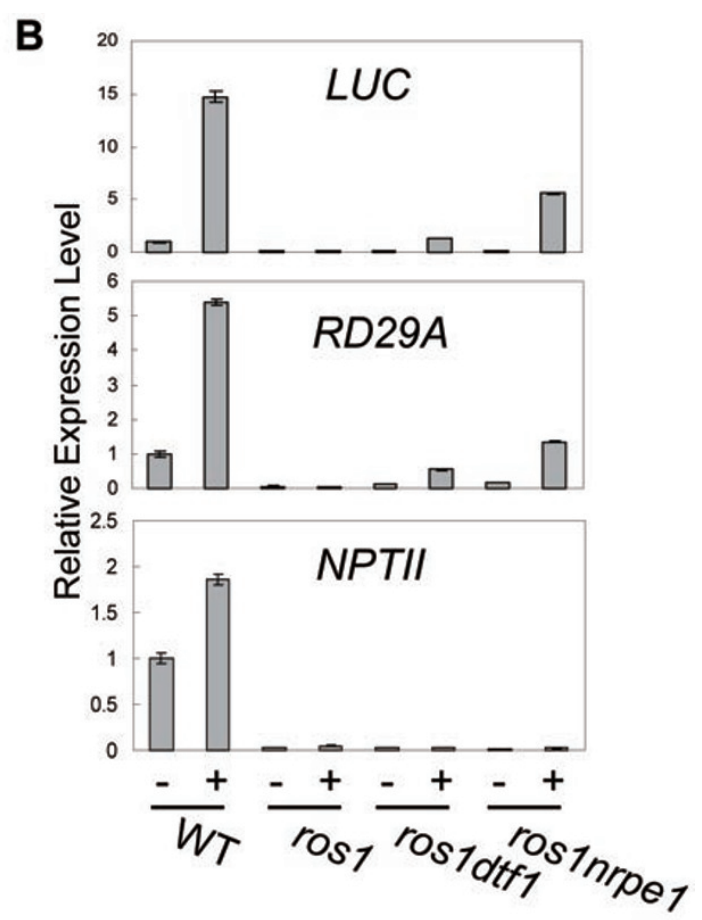

Figure 1 The $d t f 1$ mutation releases the silencing of RD29A-LUC and endogenous RD29A. (A) The dtf1 mutation releases the silencing of RD29A-LUC. The transgenic wild-type, ros1, ros1dtf1-1 and ros1nrpe1 were grown on MS medium for 2 weeks followed by treatment at $4{ }^{\circ} \mathrm{C}$ for 2 days. The treated plants were sprayed with luciferin for luminescence imaging. The same plants were grown on MS medium supplemented with $100 \mu \mathrm{g} / \mathrm{ml}$ kanamycin for 3 weeks and photographed. (B) Effect of dtf1 on expression of transgenic RD29A-LUC, endogenous RD29A and 35S-NPTII, as detected by real-time PCR. TUB4 was used as an internal control for normalization.

www.cell-research.com | Cell Research 
A

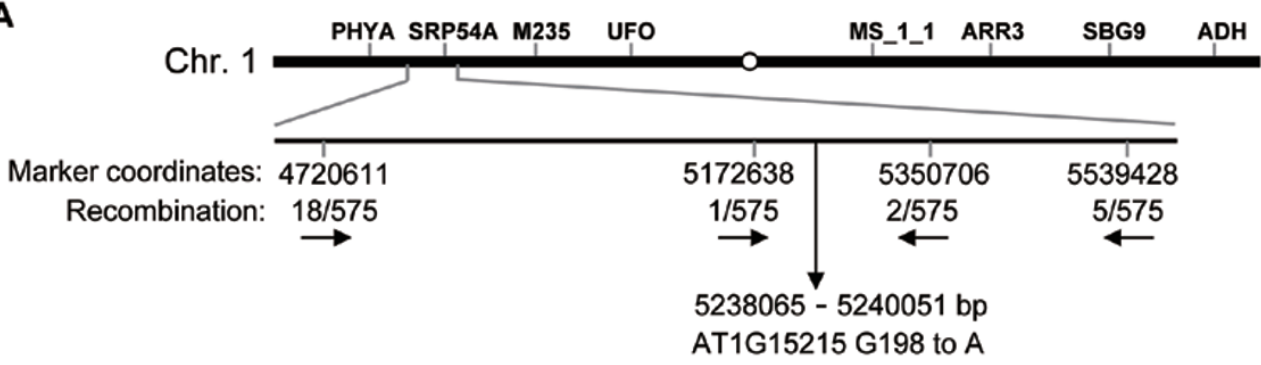

B
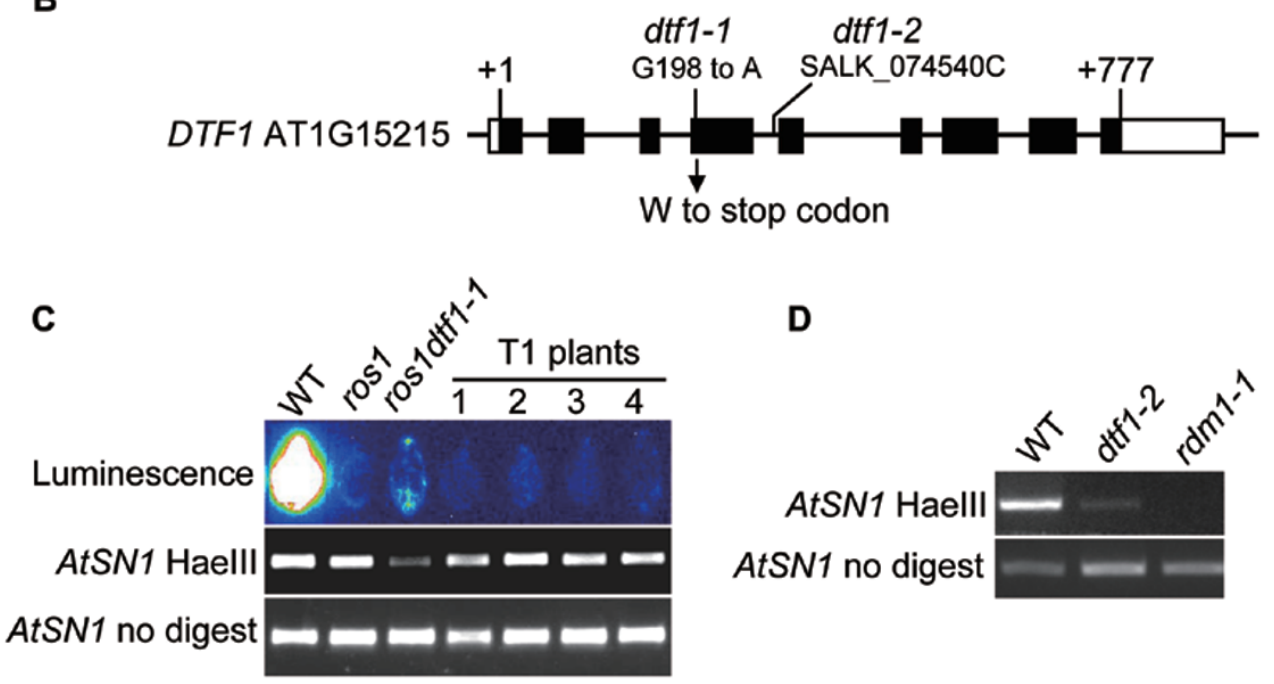

D

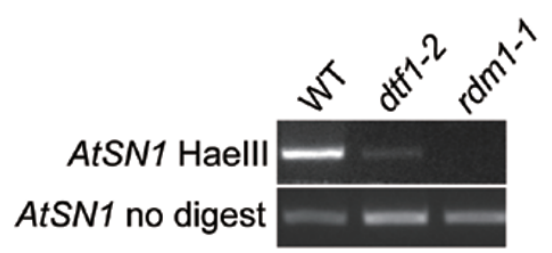

Figure 2 Map-based cloning and characterization of DTF1. (A) Map-based cloning of DTF1. The gene was located to the indicated $\sim 180 \mathrm{~kb}$ region of chromosome 1 by genetic mapping. A G-to-A mutation in the fourth exon of At1G15215 creates a premature stop codon. (B) Diagram of DTF1 and the dtf1-1 and dtf1-2 mutations. The diagram shows the DTF1 gene with the indicated positions of exons (boxes), introns (line) and open reading frame (solid boxes). The dtf1-1 and dtf1-2 mutations are labeled. (C) Complementation assay in ros1dtf1-1. The leaves from wild type, ros1, ros1dtf1-1, and four individual DTF1 transgenic T1 plants in ros1dtf1-1 were collected for luminescence imaging after treatment with $2 \% \mathrm{NaCl}$ for 4 hours. The AtSN1 methylation was tested by chop-PCR. (D) The dtf1-2 mutation reduces AtSN1 DNA methylation. Genomic DNA from the indicated genotypes was digested with Haelll, and used for amplification of AtSN1. The rdm1 mutant was used as a control.

DTF 1 encodes a putative DNA-binding transcription factor that contains a homeodomain in its N-terminal region. The homeodomain is known as the DNA-binding domain in some transcription factors. In addition to DTF1, there is a DTF1-like protein, At1G18380, encoded in the Arabidopsis genome. DTF1 and the DTF1like protein are highly similar, except that the DTF1like protein has a C-terminal extension (Supplementary information, Figure S1A). However, T-DNA insertion mutations in At1g18380 have no apparent effect on DNA methylation and transcriptional gene silencing (data not shown), suggesting that At1G18380 may have functions other than in transcriptional gene silencing.

DTF 1 encodes a plant-specific gene, which is conserved in angiosperms, gymnosperms and bryophytes (Supplementary information, Figure S1B and S1C), but not in fungi and animals. DTF1 is conserved in the Nterminal homeodomain, which may function in DNA binding. In addition, the uncharacterized domain in the $\mathrm{C}$-terminal half is also highly conserved.

DTF1 is required for the silencing of some endogenous RdDM targets and accumulation of 24-nt siRNAs

The above results showed that DTF1 is required for transgene silencing. Using semi-quantitative RT-PCR, we tested the effect of the $d t f 1-1$ mutation on the silencing of endogenous RdDM targets. The results show that the $d t f 1-1$ mutation released silencing of a subset of RdDM targets (Figure 3A). solo-LTR and AtGPl were previously identified as RdDM targets, which show a low level of expression in wild-type and ros 1 plants [45, 47]. The dtf1-1 mutation released the silencing of solo- 
$L T R$ and $A t G P 1$, while it weakly released the silencing of AtSN1A (Figure 3A). As a control, the nrpel mutation caused more substantial expression of AtSN1 A. The results suggest that DTF1 is required for the silencing of a subset of RdDM targets.

Pol V-dependent noncoding RNA transcripts are required for RdDM and transcriptional gene silencing [11]. We tested and found that the dtfl-1 mutation did not affect Pol V-dependent transcripts, while loss of the largest subunit of Pol V, NRPE1, blocked accumulation of the Pol V-dependent transcripts, including AtSN1 B and IGN5 B (Figure 3A). Previous studies showed that mutations in canonical RdDM components suppress ROS1 expression $[21,48,49]$. We found that the $d t f 1-1$ mutation also suppressed the expression of ROS1 (Figure 3A).

Accumulation of 24-nt siRNAs was detected in ros1dtf1-1 as well as in the wild type, ros 1 , and roslnrpel. 24-nt siRNAs generated from the transgenic $R D 29 \mathrm{~A}$ promoter are required for $R D 29 A-L U C$ silencing in $\operatorname{ros} 1$
[45]. RNA blot analysis showed that the level of $R D 29 \mathrm{~A}$ promoter siRNAs was reduced in ros 1dtf1-1 and ros1nrpel (Figure 3B). The result suggests that the role of DTF1 in RD29A-LUC trangene silencing is related to siRNA-directed DNA methylation and transcriptional silencing. The accumulation of 24-nt siRNAs was also reduced in rosldtf1-1 at some endogenous RdDM targets, including AtSN1, solo-LTR and 5S rDNA, whereas the siRNAs from some other genomic loci (AtGP1, AtMU1, Cluster4, and siRNA02) were not affected by the dtf1 mutation (Figure 3B). The pattern of siRNA accumulation in rosldtf1-1 was very similar to that in ros 1nrpe1, although the $d t f 1$ mutation affected siRNA accumulation to a greater extent than nrpel (Figure 3B). Neither transacting siRNA255 nor microRNA171 was affected in ros 1dtf1-1 and ros 1nrpel (Figure 3B), supporting that DTF 1 specifically functions in 24-nt siRNA-directed transcriptional gene silencing.
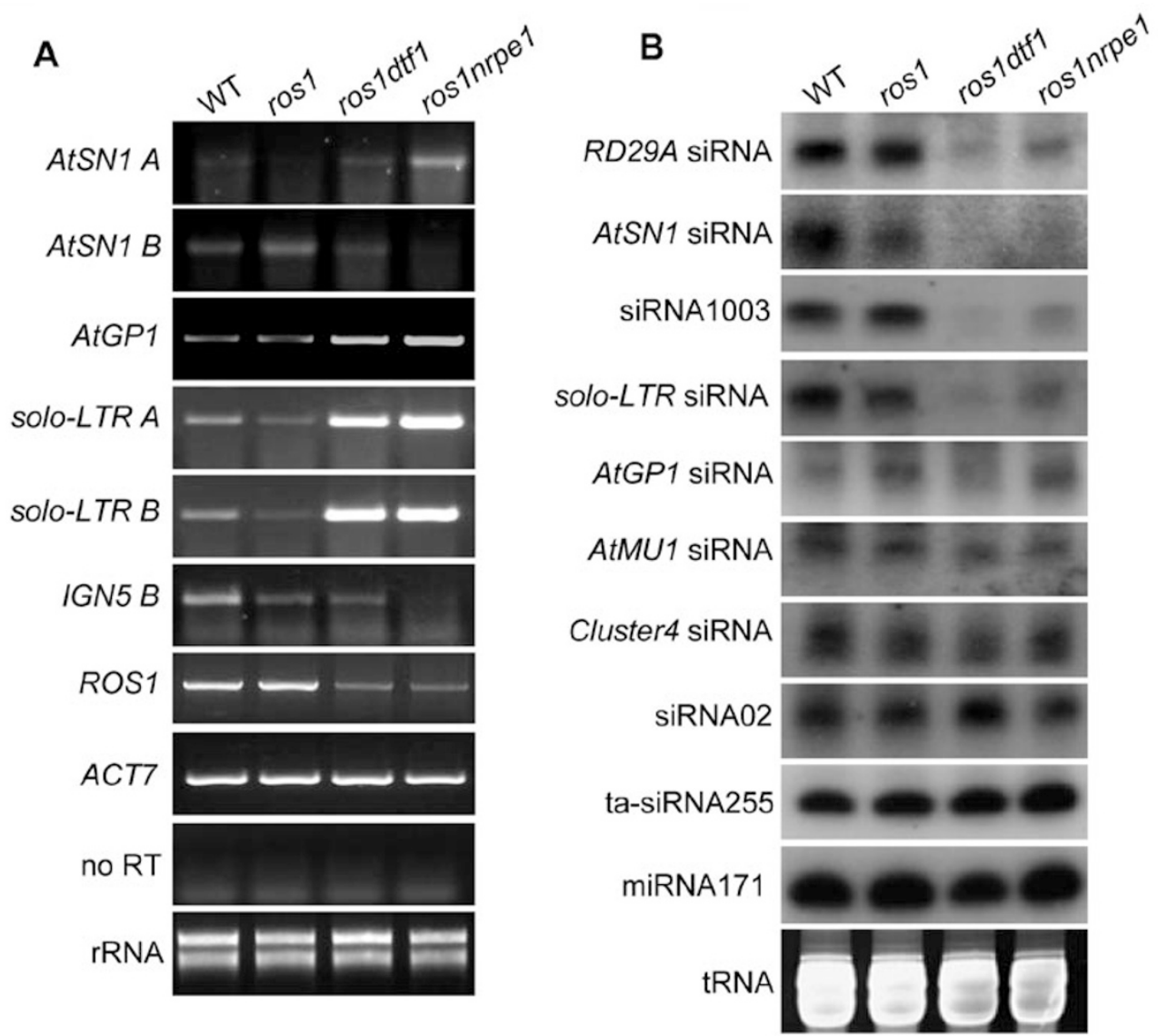

Figure 3 Effect of $d t f 1$ mutation on transcriptional gene silencing and small RNA accumulation. (A) Effect of $d t f 1$ on the transcript levels of endogenous RdDM targets was determined by semi-quantitative RT-PCR. ACT7 was used as an internal control. No RT amplification indicates that there was no DNA contamination in RNA samples. (B) Effect of $d t f 1$ on accumulation of small RNAs. The levels of 24-nt siRNAs, 21-nt ta-siRNA255 and miRNA171 were tested in the indicated genotypes. The ethidium bromide-stained small RNA gel was used as a loading control. 
A

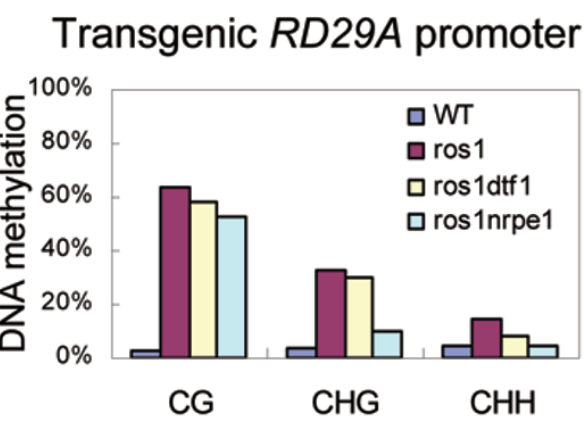

C

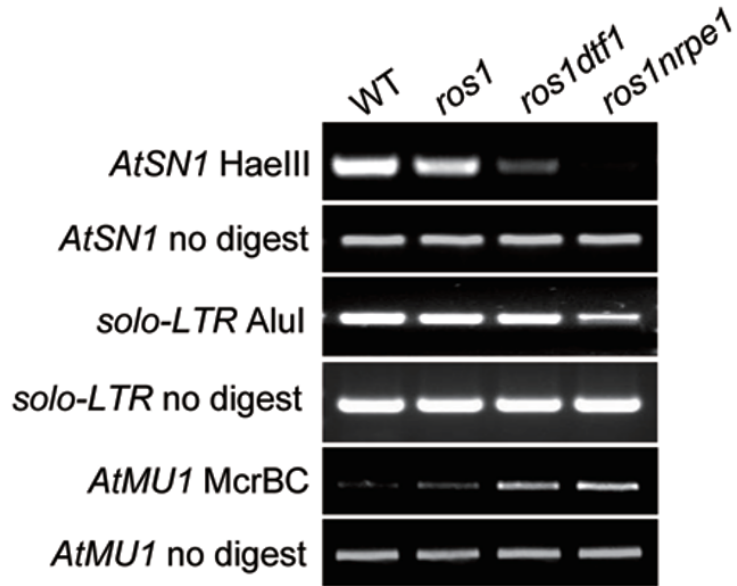

$\mathbf{E}$

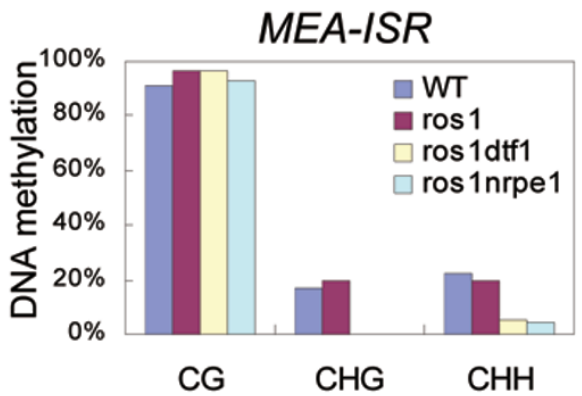

B

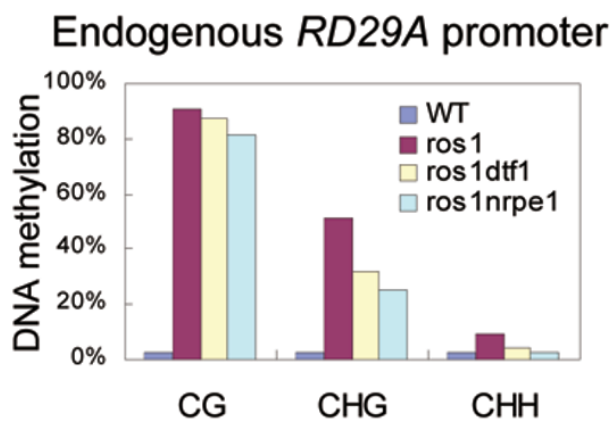

D

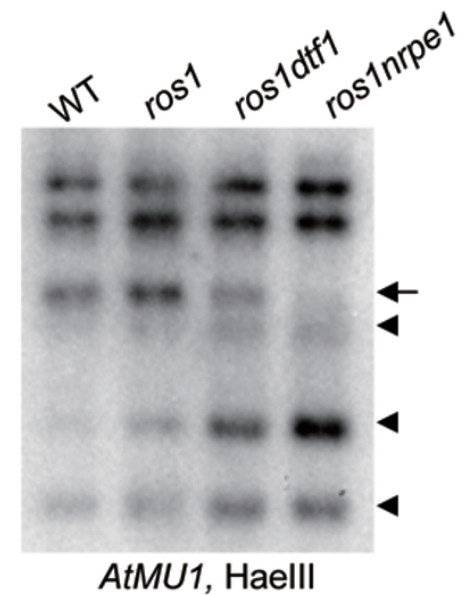

$\mathbf{F}$

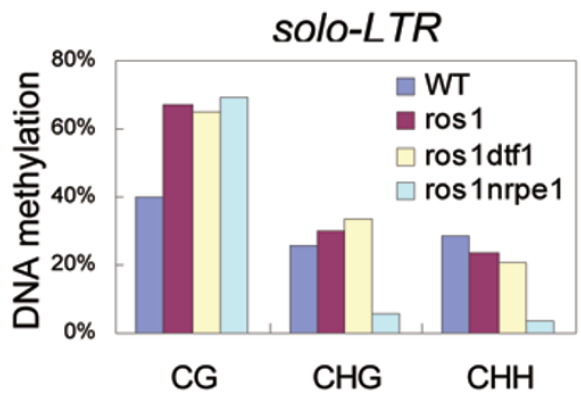

Figure 4 Effect of $d t f 1$ on DNA methylation. (A, B) The effect of $d t f 1$ on the promoter DNA methylation of RD29A-LUC (A) and endogenous $R D 29 A$ (B) was determined by bisulfite sequencing. The percentage of methylated cytosine in different cytosine contexts is shown. (C) The DNA methylation level of AtSN1, solo-LTR and AtMU1 was determined by chop-PCR. The DNA methylation-sensitive restriction enzymes Haelll and Alul, and DNA mehylation-insensitive enzyme McrBC were used for DNA methylation analysis as indicated. (D) The effect of dtf1 on AtMU1 DNA methylation was detected by Southern blot analysis. Genomic DNA from the indicated genotypes was digested by Haelll followed by Southern hybridization. (E and F) The DNA methylation status of MEA-ISR and solo-LTR was determined by bisulfite sequencing in the indicated genotypes.

DTF1 is required for DNA methylation at some endogenous RdDM targets

To test whether the suppression of transcriptional gene silencing in ros $1 \mathrm{dtf1-1}$ is correlated with a reduction in DNA methylation, we analyzed the DNA methylation status at the RD29A-LUC transgene, endogenous
$R D 29 A$ and several other endogenous RdDM targets. Consistent with our previous results [41], the DNA methylation level of both transgenic and endogenous $R D 29 \mathrm{~A}$ promoters was very low in wild type, and the methylation in all cytosine contexts $(\mathrm{CG}, \mathrm{CHG}, \mathrm{CHH})$ was substantially increased in ros 1 (Figure 4A and 4B). 
The DNA methylation level was well correlated with the silencing of RD29A-LUC transgene and endogenous $R D 29 A$ gene. We found that the $R D 29 A$ promoter DNA methylation at non-CG sites was reduced in rosldtfl-1, as in ros 1nrpel (Figure 4A and 4B). The results further suggest that DTF1 functions in RdDM. Consistent with the weak reactivation of $A t S N 1$ in $d t f 1$ mutants (Figure $3 \mathrm{~A}$ ), the AtSN1 DNA methylation level was partially reduced by the $d t f 1$ mutation (Figure $4 \mathrm{C}$ ). The DNA methylation of the endogenous RdDM target AtMU1 was reduced in rosldtf1-1 as well as in ros 1nrpel (Figure 4C and 4D), although siRNA accumulation at the locus was not affected by $d t f 1$ or nrpe1 (Figure 3B). These results suggest that like NRPE1, DTF1 functions downstream of siRNA generation to mediate DNA methylation and transcriptional gene silencing. As in roslnrpe1, non-CG DNA methylation at MEA-ISR was reduced in ros Idtf1-1 (Figure 4E). Taken together, the results suggest that DTF1 is a new component of RdDM, and it functions in the DNA methylation and transcriptional silencing of a subset of RdDM targets, including AtMU1 and MEA-ISR.

As the silencing of solo-LTR is substantially released in ros1dtf1-1, we tested whether the DNA methylation of solo-LTR is reduced in the mutant by bisulfite sequencing. The results show that the DNA methylation of solo-LTR was clearly reduced at $\mathrm{CHG}$ and $\mathrm{CHH}$ sites in ros 1nrpe1, but not in ros 1dtf1-1 (Figure 4F). This is in contrast to the silencing of solo-LTR, which was released to similar levels in ros 1dtf1-1 and ros 1nrpe1 (Figure $3 \mathrm{~A})$. Futhermore, the DNA methylation of $5 \mathrm{~S}$ rDNA repeats was also not affected by the $d t f 1$ mutations in ros $1 d t f 1-1$ as well as in $d t f 1-2$, whereas the DNA methylation was significantly reduced in the canonical RdDM mutants nrpdl and nrpel (Figure 5A and 5B). However, although the DNA methylation of both solo-LTR and $5 \mathrm{~S}$ rDNA was not altered by $d t f 1$, the accumulation of solo-LTR siRNAs and siRNA1003 (from 5S rDNA) was substantially reduced by $d t f 1$. These results suggest that the function of DTF1 in siRNA-mediated transcriptional gene silencing at solo-LTR and 5S rDNA is independent of DNA methylation.

\section{Discussion}

In the canonical RdDM pathway, all previously characterized RdDM components are required for both the

\section{A}

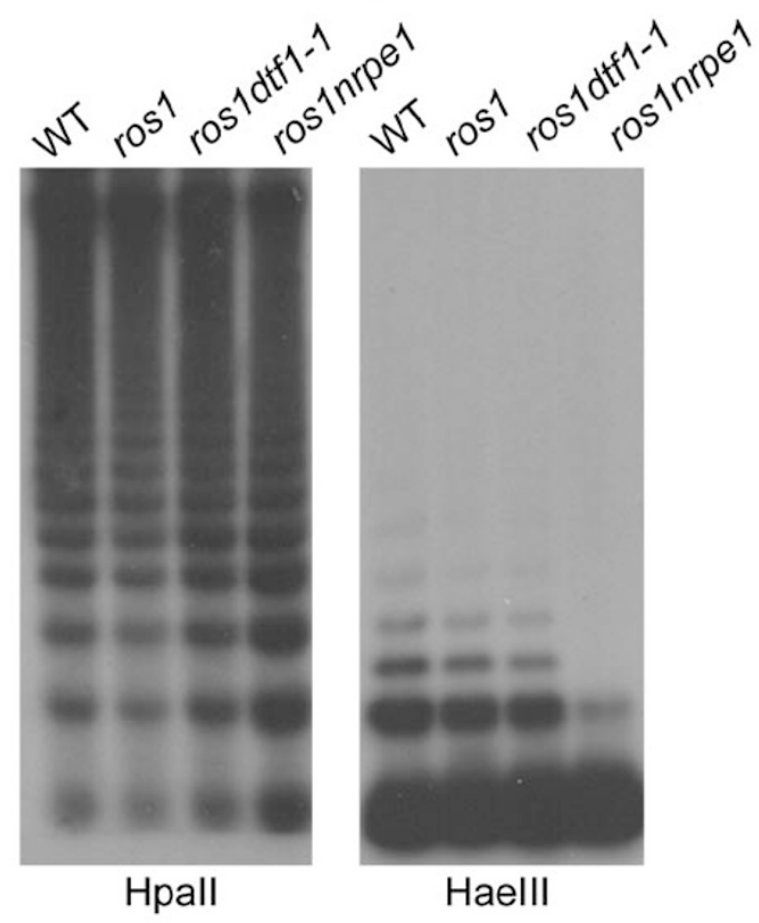

B

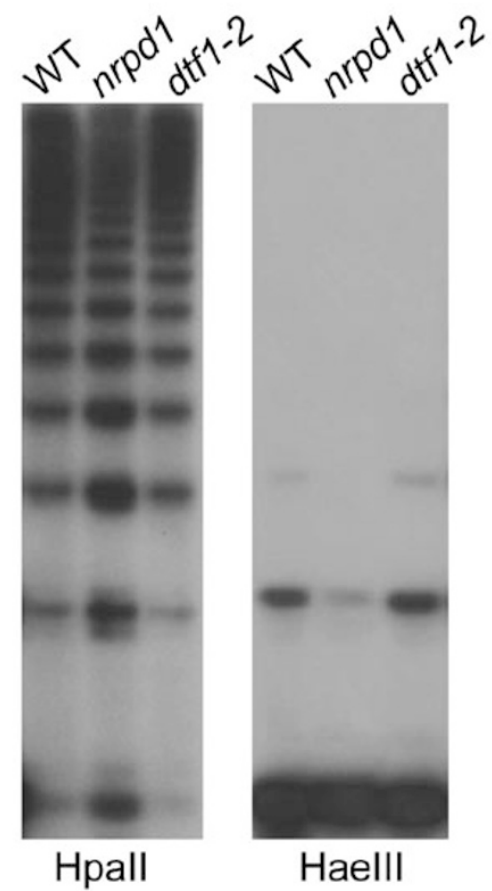

Figure 5 Effect of $d t f 1$ mutations on $5 S$ rDNA methylation. Genomic DNA from the indicated genotypes was digested with Hpall (for CG and CHG methylation) and Haelll (for $\mathrm{CHH}$ methylation), and used for Southern blot analysis. The effect of $d t f 1$ mutations on the DNA methylation of 5S rDNA repeats was tested in ros1dtf1-1 (A) and dtf1-2 (B), respectively. 
establishment of DNA methylation and for transcriptional gene silencing at target loci. solo-LTR is one of the RdDM targets, and was first discovered by the Matzke group [47]. In nrpd1, nrpe1, rdr2 and $d r d 1$, the DNA methylation of solo-LTR is substantially reduced, resulting in the release of transcriptional gene silencing at solo-LTR in these mutants $[11,47,50]$. However, different from the canonical RdDM components, we found that DTF1 is dispensable for DNA methylation at solo$L T R$, although it is required for siRNA accumulation and transcriptional gene silencing at this locus. It is worth noticing that solo-LTR is a rather unusual RdDM target in that the repressive histone mark $\mathrm{H} 3 \mathrm{~K} 27$ monomethylation (H3K27me1) is substantially reduced at solo-LTR by mutations of the canonical RdDM components, whereas the mark is not changed in the mutants at the other tested RdDM targets [47]. The dependence of H3K27me1 on $\mathrm{RdDM}$ at solo-LTR may reflect a special chromatin structure or DNA sequence specificity of the locus, which may be absent at the other tested RdDM target loci. The recently characterized Arabidopsis H3K27 monmethyltransferases, ATXR5 and ATXR6, are involved in transcriptional silencing of transposable elements and other repetitive DNA sequences [51]. The silencing status of some RdDM targets such as solo-LTR may require both DNA methylaion and H3K27me1 catalyzed by ATXR5/ ATXR6. In this study, we found that the dtfl mutation suppresses silencing of solo-LTR without affecting DNA methylation, suggesting that at this locus DTF1 may function downstream of DNA methylation. As a homeodomain-containing transcription factor, DTF1 may recognize the special chromatin structure or DNA sequence of solo-LTR to help siRNAs guide H3K27 monomethylation and transcriptional silencing. At the other RdDM targets tested, DTF1 is required for both DNA methylation and silencing, just like the canonical RdDM components. One possibility is that DTF1 may be involved in siRNAdirected heterochromatic histone modifications, such as H3K9 methylation and H3K27 methylation, which may occur upstream (e.g., H3K9 methylation) or downstream (e.g., H3K27 methylation) of DNA methylation in the RdDM pathway. Regardless of the exact mechanism of function, our results suggest that DTF1 is an atypical RdDM component that mediates transcriptional gene silencing in both DNA methylation-dependent and -independent manners.

Our small RNA blot analysis showed that DTF1 is required for accumulation of 24-nt siRNAs at some RdDM targets but has no effect on the others. The siRNA pattern in ros 1dtfl-1 appears the same as that in roslnrpel, suggesting that like Pol V, DTF1 may not be involved in the initial biogenesis of siRNAs, but may affect the ac- cumulation of some siRNAs indirectly by influencing the heterochromatic marks of the loci. It is interesting that $d t f 1$ affects siRNA accumulation to a greater extent than nrpe1, although the effect of $d t f 1$ on DNA methylation is less or even undetectable. The disparaging effects of the two mutations (dtfl and nrpel) on siRNA accumulation and DNA methylation hint at unknown complexities and mechanisms of RdDM. During the preparation of this manuscript, Law et al. [52] reported that SHH1 is required for DNA methylation, and associates with Pol IV based on affinity purification of NRPD1. SHH1, which is the same protein as DTF1, was suggested to function in siRNA biogenesis as a transcription factor for Pol IV. In the Law et al. study, RDR2, CLSY1 and RDM4 were also shown to be associated with NRPD1. RDR2 is abundant in the affinity-purified NRPD1-containing complex, and likely functions together with Pol IV to produce siRNAs. The levels of CLSY1, RDM4 and SHH1 in the purified NRPD1-containing complex were very low [52]. Moreover, RDM4 is known to be a Pol V-interacting protein required for the generation of Pol V-dependent transcripts [21]. These observations suggest that CLSY1, RDM4 and SHH1 are unlikely to be core components of the NRPD1-containing complex. Our results show clearly that unlike Pol IV, DTF1 is only required for the accumulation of siRNAs at a subset of RdDM targets. Importantly, we discovered that $d t f 1$ reduces siRNA accumulation and releases silencing at solo-LTR without affecting DNA methylation. In addition, we found that $d t f 1$ reduces siRNA accumulation from $5 \mathrm{~S}$ rDNA repeats but has no apparent effect on DNA methylation of the rDNA. These surprising observations suggest that besides causing de novo DNA methylation, siRNAs may also guide the establishment of other silencing epigenetic marks such as heterochromatic histone modifications. DTF1 may be involved in this later function of siRNAs in the RdDM pathway. Previous studies have found that some Pol IV-dependent 24-nt siRNAs do not cause DNA methylation [53]. It would be of great interest to determine the DNA methylation-independent chromatin functions of the siRNAs and the role of DTF1 in these functions.

\section{Materials and Methods}

\section{Plant materials, mutant screening and cloning}

The wild-type $\mathrm{C} 24$ and ros 1 mutant carry a homozygous RD29A promoter-driven luciferase transgene [54]. The ros 1 mutant with RD29-LUC transgene was mutagenized with ethyl methanesulfonate. Suppressors of ros 1 were screened by luminescence imaging after the mutagenized M2 plants were cold-treated for 2 days at $4{ }^{\circ} \mathrm{C}$. The selected plants were transplanted into soil, and the luminescence phenotype was confirmed in adult plants as previously described [45]. Mutants of interest were crossed to 
the ros 1 mutant in the Col-0 backcrossed for map-based cloning. High-throughput sequencing (Illumina) was carried out to detect the mutations in the mapped genomic interval. For complementation assay, the full-length $D T F 1$ genomic sequence was amplified, and cloned into the binary vector pCAMBIA1300. The DTF1 construct was transformed into ros 1dtf1-1 by the Agrobacterium strain GV3101. The transgenic T1 plants were tested for phenotypic complementation.

Analysis of RNA transcript level and small RNA accumulation

Total RNA was isolated from 2-week-old seedlings on MS plates as described previously [45]. For RT-PCR, the sequences of primers are listed in Supplementary information, Table S1. After contaminating DNA was removed by DNase, total RNA was used for RT-PCR. For RT-PCR of protein-encoding genes, oligo$\mathrm{dT}$ was used as the reverse primer to synthesize the first-stranded cDNA. RT-PCR of transposable elements and other DNA repeat sequences was carried out by a previously described one-step RTPCR protocol [11]. Briefly, the PCR condition is $95{ }^{\circ} \mathrm{C}$ for $5 \mathrm{~min}$ followed by $28-40$ amplification cycles $\left(95^{\circ} \mathrm{C}\right.$ for $30 \mathrm{~s}, 56^{\circ} \mathrm{C}$ for $30 \mathrm{~s}, 72{ }^{\circ} \mathrm{C}$ for $\left.1 \mathrm{~min}\right)$. The constitutively expressed ACT7 was used as an internal control. For small RNA analysis, small RNA was extracted as described previously [45], and was separated on a $15 \%$ polyacrylamide gel at $200 \mathrm{~V}$ for $3 \mathrm{~h}$. The small RNA was electrotransfered to Hybond- $\mathrm{N}^{+}$membranes (Amersham). The probes for small RNA hybridization are $\left[\gamma-{ }^{32} \mathrm{P}\right]$ ATP-labeled DNA oligonucleotides or $\left[\alpha-{ }^{32} \mathrm{P}\right] \mathrm{dCTP}$-labeled amplified DNA. The small RNA hybridization was carried out in PerfectHyb buffer (Sigma) at $38^{\circ} \mathrm{C}$. The DNA primer and probe sequences are listed in Supplementary information, Table S1.

\section{DNA methylation assay}

The DNA methylation level was detected by chop-PCR, Southern blot analysis, or bisulfite sequencing. For chop-PCR, the genomic DNA was digested with DNA methylation-sensitive (HaeIII, $A l u \mathrm{I}$, and $M s p \mathrm{I})$ and -insensitive $(M c r B C)$ restriction enzymes, and used for amplification of target DNA sequences. For Southern blot analysis, $5 \mu \mathrm{g}$ of genomic DNA was digested with indicated DNA methylation-sensitive enzymes, and resolved on $1.2 \%$ agarose gel at $40 \mathrm{~V}$ overnight, followed by a routine Southern blotting method. For bisulfite sequencing, $2 \mu \mathrm{g}$ of genomic DNA was converted and purified with EpiTect Bisulfite Kit (Qiagen). The treated DNA was used for amplification and cloning. At least 10 individual clones were sequenced for each sample. The primers are described in Supplementary information, Table S1.

\section{Acknowledgments}

We thank the National Institute of Biological Sciences sequencing center for its excellent technical support on high-throughput DNA sequencing and analysis. This work is supported by a 973 program grant from the Chinese Ministry of Science and Technology (2011CB812600 to X-J H) and US National Institutes of Health grant (R01GM070795 to J-K Z).

\section{References}

1 Xie Z, Johansen L K, Gustafson A M, et al. Genetic and functional diversification of small RNA pathways in plants. PLoS
Biol 2004; 2:E104

2 Kanno T, Huettel B, Mette MF, et al. Atypical RNA polymerase subunits required for RNA-directed DNA methylation. Nat Genet 2005; 37:761-765.

3 Herr AJ, Jensen MB, Dalmay T, Baulcombe DC. RNA polymerase IV directs silencing of endogenous DNA. Science 2005; 308:118-120.

4 Law JA, Jacobsen SE. Establishing, maintaining and modifying DNA methylation patterns in plants and animals. Nature Rev Genet 2010; 11:204-220.

5 He XJ, Chen T, Zhu JK. Regulation of and function DNA methylation in plants and animals. Cell Res 2011; 21:442-465.

6 Matzke MA, Birchler JA. RNAi-mediated pathways in the nucleus. Nat Rev Genet 2005; 6:24-35.

7 Mosher RA, Melnyk CW, Kelly KA, et al. Uniparental expression of PolIV-dependent siRNAs in developing endosperm of Arabidopsis. Nature 2009; 460:283-286.

8 Hsieh TF, Ibarra CA, Silva P, et al. Genome-wide demethylation of Arabidopsis endosperm. Science 2009; 324:1451-1454.

9 Gehring M, Bubb KL, Henikoff S. Extensive demethylation of repetitive elements during seed development underlies gene imprinting. Science 2009; 324:1447-1451.

10 Ito H, Gaubert H, Bucher E, et al. An siRNA pathway prevents transgenerational retrotransposition in plants subjected to stress. Nature 2011; 472:115-119.

11 Wierzbicki AT, Haag JR, Pikaard CS. Non-coding transcription by RNA polymerase Pol IVb/Pol V mediates transcriptional silencing of overlapping and adjacent genes. Cell 2008; 135:635-648.

12 Pontier D, Yahubyan G, Vega D, et al. Reinforcement of silencing at transposons and highly repeated sequences requires the concerted action of two distinct RNA polymerases IV in Arabidopsis. Genes Dev 2005; 19:2030-2040.

13 Pontes O, Li C F, Nunes PC, et al. The Arabidopsis chromatinmodifying nuclear siRNA pathway involves a nucleolar RNA processing center. Cell 2006; 126:79-92.

14 Li CF, Pontes O, El-Shami M, et al. An ARGONAUTE4-containing nuclear processing center colocalized with Cajal bodies in Arabidopsis thaliana. Cell 2006; 126:93-106.

15 Kanno T, Mette MF, Kreil DP, et al. Involvement of putative SNF2 chromatin remodeling protein DRD1 in RNA-directed DNA methylation. Curr Biol 2004; 14:801-805.

16 Kanno T, Bucher E, Daxinger L, et al. A structural-maintenance-of chromosomes hinge domain-containing protein is required for RNA-directed DNA methylation. Nat Genet 2008; 40:670-675.

17 Law JA, Ausin I, Johnson LM, et al. A protein complex required for polymerase $\mathrm{V}$ transcripts and RNA-directed DNA methylation in Arabidopsis. Curr Biol 2010; 20:951-956.

18 Gao Z, Liu HL, Daxinger L, et al. An RNA polymerase II- and AGO4-associated protein acts in RNA-directed DNA methylation. Nature 2010; 465:106-109.

19 He XJ, Hsu YF, Zhu S, et al. An effector of RNA-directed DNA methylation in Arabidopsis is an ARGONAUTE 4- and RNA-binding protein. Cell 2009; 137:498-508.

20 Bies-Etheve N, Pontier D, Lahmy S, et al. RNA-directed DNA methylation requires an AGO4-interacting member of the SPT5 elongation factor family. EMBO Rep 2009; 10:649-654.

$21 \mathrm{He}$ XJ, Hsu YF, Zhu S, et al. A conserved transcriptional regu- 
lator is required for RNA-directed DNA methylation and plant development. Genes Dev 2009; 23:2717-2722.

22 Kanno T, Bucher E, Daxinger L, et al. RNA-directed DNA methylation and plant development require an IWR1-type transcription factor. EMBO Rep 2010; 11:65-71.

23 Cao X, Jacobsen SE. Role of the Arabidopsis DRM methyltransferases in de novo DNA methylation and gene silencing. Curr Biol 2002; 12:1138-1144.

24 Naumann U, Daxinger L, Kanno T, et al. Genetic evidence that DNA methyltransferase DRM2 has a direct catalytic role in RNA-directed DNA methylation in Arabidopsis thaliana. Genetics 2011; 187:977-979.

25 Greenberg MV, Ausin I, Chan SW, et al. Identification of genes required for de novo DNA methylation in Arabidopsis. Epigenetics 2011; 6:344-354.

26 Henderson IR, Deleris A, Wong W, et al. The de novo cytosine methyltransferase DRM2 requires intact UBA domains and a catalytically mutated paralog DRM3 during RNA-directed DNA methylation in Arabidopsis thaliana. PLoS Genet 2010; 6:e1001182.

27 Moazed D. Small RNAs in transcriptional gene silencing and genome defence. Nature 2009; 457:413-420.

28 Motamedi MR, Verdel A, Colmenares SU, et al. Two RNAi complexes, RITS and RDRC, physically interact and localize to noncoding centromeric RNAs. Cell 2004; 119:789-802.

29 Verdel A, Jia S, Gerber S, et al. RNAi-mediated targeting of heterochromatin by the RITS complex. Science 2004; 303:672676.

30 Debeauchamp JL, Moses A, Noffsinger VJ, et al. Chp1-Tas3 interaction is required to recruit RITS to fission yeast centromeres and for maintenance of centromeric heterochromatin. Mol Cell Biol 2008; 28:2154-2166.

31 Nakayama J, Rice JC, Strahl BD, Allis CD, Grewal SI. Role of histone H3 lysine 9 methylation in epigenetic control of heterochromatin assembly. Science 2001; 292:110-113.

32 Cao X, Jacobsen SE. Control of CpNpG DNA methylation by the KRYPTONITE histone H3 methyltransferase. Nature 2002; 416:556-560.

33 Lindroth AM, Shultis D, Jasencakova Z, et al. Dual histone $\mathrm{H} 3$ methylation marks at lysines 9 and 27 required for interaction with CHROMOMETHYLASE3. EMBO J 2004; 23:42864296.

34 Johnson LM, Bostick M, Zhang X, et al. The SRA methylcytosine-binding domain links DNA and histone methylation. Curr Biol 2007; 17:379-384.

35 Yokthongwattana $\mathrm{C}$, Bucher E, Caikovski M, et al. MOM1 and Pol-IV/V interactions regulate the intensity and specificity of transcriptional gene silencing. EMBO J 2010; 29:340-351.

36 Numa H, Kim JM, Matsui A, et al. Transduction of RNAdirected DNA methylation signals to repressive histone marks in Arabidopsis thaliana. EMBO J 2010; 29:352-362.

37 Amedeo P, Habu Y, Afsar K, Mittelsten Scheid O, Paszkowski J. Disruption of the plant gene MOM releases transcriptional silencing of methylated genes. Nature 2000; 405:203-206.

38 Zhou Y, Zhang J, Lin H, Guo G, Guo Y. MORPHEUS' MOLECULE1 is required to prevent aberrant RNA transcriptional read-through in Arabidopsis. Plant Physiol 2010; 154:12721280 .
39 Caikovski M, Yokthongwattana C, Habu Y, et al. Divergent evolution of CHD3 proteins resulted in MOM1 refining epigenetic control in vascular plants. PLoS Genet 2008; 4:e1000165.

40 Petty TJ, Nishimura T, Emamzadah S, et al. Expression, crystallization and preliminary X-ray diffraction analysis of the CMM2 region of the Arabidopsis thaliana Morpheus' molecule 1 protein. Acta Crystallogr Sect F Struct Biol Cryst Commun 2010; 66: 916-918.

41 Gong Z, Morales-Ruiz T, Ariza RR, et al. ROS1, a repressor of transcriptional gene silencing in Arabidopsis, encodes a DNA glycosylase/lyase. Cell 2002; 111:803-814.

42 Agius F, Kapoor A, Zhu JK. Role of the Arabidopsis DNA glycosylase/lyase ROS1 in active DNA demethylation. Proc Natl Acad Sci USA 2006; 103:11796-11801.

43 Zhu J, Kapoor A, Sridhar VV, et al. The DNA glycosylase/ lyase ROS1 functions in pruning DNA methylation patterns in Arabidopsis. Curr Biol 2007; 17:54-59.

44 Penterman J, Zilberman D, Huh JH, et al. DNA demethylation in the Arabidopsis genome. Proc Natl Acad Sci USA 2007; 104:6752-6757.

$45 \mathrm{He}$ XJ, Hsu YF, Pontes O, et al. NRPD4, a protein related to the RPB4 subunit of RNA polymerase II, is a component of RNA polymerases IV and V and is required for RNA-directed DNA methylation. Genes Dev 2009; 23:318-330.

46 Kapoor A, Agarwal M, Andreucci A, et al. Mutations in a conserved replication protein suppress transcriptional gene silencing in a DNA-methylation-independent manner in Arabidopsis. Curr Biol 2005; 15:1912-1918.

47 Huettel B, Kanno T, Daxinger L, et al. Endogenous targets of RNA-directed DNA methylation and Pol IV in Arabidopsis. EMBO J 2006; 25:2828-2836.

48 Mathieu O, Reinders J, Caikovski M, Smathajitt C, Paszkowski J. Transgenerational stability of the Arabidopsis epigenome is coordinated by CG methylation. Cell 2007; 130:851-862.

49 Penterman J, Uzawa R, Fischer RL. Genetic interactions between DNA demethylation and methylation in Arabidopsis. Plant Physiol 2007; 145:1549-1557.

50 Zheng B, Wang Z, Li S, et al. Intergenic transcription by RNA polymerase II coordinates Pol IV and Pol V in siRNA-directed transcriptional gene silencing in Arabidopsis. Genes Dev 2009; 23:2850-2860.

51 Jacob Y, Feng S, LeBlanc CA, et al. ATXR5 and ATXR6 are H3K27 monomethyltransferases required for chromatin structure and gene silencing. Nat Struct Mol Biol 2009; 16:763-768.

52 Law JA, Vashisht AA, Wohlschlegel JA, Jacobsen SE. SHH1, a homeodomain protein required for DNA methylation, as well as RDR2, RDM4, and chromatin remodeling factors, associate with RNA polymerase IV. PLoS Genet 2011; 7:e1002195.

53 Mosher RA, Schwach F, Studholme D, Baulcombe DC. Po$1 \mathrm{IVb}$ influences RNA-directed DNA methylation independently of its role in siRNA biogenesis. Proc Natl Acad Sci USA 2008; 105:3145-3150.

54 Ishitani M, Xiong L, Stevenson B, Zhu JK. Genetic analysis of osmotic and cold stress signal transductionin Arabidopsis: Interactions and convergence of abscisic acid-dependent and abscisic acid-independent pathways. Plant Cell 1997; 9:19351949.

(Supplementary information is linked to the online version of the paper on the Cell Research website.) 